\title{
Physical activity and features of the environment in which school children grow up as low mood determinants
}

\author{
Dorota Kleszczewska', Jadwiga Siedlecka², Joanna Mazur ${ }^{3}$ \\ 'Foundation of the Institute of Mother and Child, Warsaw, Poland \\ 2Department of Work Physiology and Ergonomics, Nofer Institute of Occupational Medicine, Lodz, Poland \\ ${ }^{3}$ Department of Child and Adolescent Health, Institute of Mother and Child, Warsaw, Poland
}

\section{ABSTRACT}

Introduction: Reduced wellbeing and low mood also apply to young people and may lead to serious mental problems.

Aim of the study: The purpose of the study was to analyse the prevalence of depression threat symptoms depending on the level of physical activity of young people, taking into account environmental factors and the quality of social relations.

Material and methods: The survey conducted within the framework of the last round of international Health Behaviour in School-aged Children (HBSC) studies in the academic year 2017/2018 covered 5225 students aged 11-15 years from all over Poland. The mental wellbeing of young people measured by the WHO- 5 screen test was the dependent variable. Sixteen independent variables were taken into account, in blocks related to the assessment of demographic features, physical activity, social and economic position, and social relations. Results: The study demonstrated that $14.2 \%$ of respondents had depression threat symptoms. After a multidimensional analysis the main risk factors were low level of family support $(\mathrm{OR}=3.20)$, maladjustment to school $(\mathrm{OR}=2.79)$, inability to cope in social situations $(\mathrm{OR}=2.52)$, and low physical activity $(\mathrm{OR}=2.15)$. Factors associated with living conditions do not have a direct effect on depression risk but moderate the impact of physical activity on its symptoms.

Conclusions: The protective effect of physical activity on the mental health of teenagers is maintained after taking into account several other predictors of reduced wellbeing. Factors associated with living conditions do not have a direct influence on depression risk but moderate the effect of physical activity on its symptoms. The protective effect of physical activity is particularly visible in single-parent families, in poor families, and in large cities. It is important to design programs providing equal opportunities to children growing up in a less favourable environment by providing young people from the poorest families with the ability to participate in free of charge sports activities, particularly team sports.

\section{KEY WORDS:}

well-being, adolescents, physical activity, environmental features.

\section{ADDRESS FOR CORRESPONDENCE:}

Dorota Kleszczewska, Foundation of the Institute of Mother and Child, 17a Kasprzaka St., 01-211 Warsaw,

Poland, ORCID: 0000-0001-7523-8628, e-mail: dorota.kleszczewska@imid.med.pl 


\section{INTRODUCTION}

\section{LOW MOOD AMONG TEENAGERS - AN INCREASINGLY FREQUENT PROBLEM AFFECTING YOUNG PEOPLE}

Mental health problems associated with broadly understood lowered or depressed mood increasingly affect children and young people [1]. Low mood may consequently lead to more serious mental problems, even to depression, which is too rarely correctly diagnosed, while its first symptoms may pass unnoticed by the child's family and friends [2]. Primary health care doctors also have problems with early detection of depression symptoms in children and adolescents. It should be remembered that many young people with mental disorders never actually reach the doctor due to the general opinion that mental problems do not affect children [3].

Low mental wellbeing affects the everyday functioning of children and young people, although this is a mutual association. Difficulties in peer and family relationships appear, accompanied by weaker cognitive development and problems with studying [4].

The appearance of mood disorders in adolescence must be seen as a complex process with a variety of underlying causes. They may have biological grounds, such as hormonal changes associated with puberty and genetic determinants, as well as social causes [5]. It has been demonstrated that young people who have undergone a recent traumatic experience are more likely to suffer from low mood. These symptoms are also more frequent among teenagers suffering from chronic diseases.

Persons with mood disorders or mental problems try to supress negative emotions and look for ways to improve their wellbeing. Young people are more likely to start smoking tobacco [6] and reach for other psychoactive substances, including alcohol. So-called depressive individuals also frequently experience nutrition disorders. Excessive control of body mass may appear, a tendency to under-eat as well as to "overeat their problems", which consequently leads to overweight or obesity [7].

When analysing the mental problems of young people, the differences associated with gender must be remembered, which change with age. In early childhood gender is insignificant, differences unfavourable for girls becoming visible in early adolescence. Teenage girls tend to have lower moods among others due to problems with accepting their body, which changes during puberty. Girls increasingly use community portals and communicators, as a result of which they are exposed to negative opinions about themselves and all forms of cyberbullying [8]. Use of contemporary media may also have the opposite effect, i.e. it may counter mood disorders by facilitating contact between people, making new friends, or talking to friends and family [9].

The unfavourable influence of the environment in which young people grow up may be an additional risk factor which increases the frequency of mood disorder symptoms. The level of family affluence and social capital of the neighbourhood in which teenagers grow up and study are worth mentioning [10]. On the other hand, physical activity may play a so-called protective role, and programs which promote it may lead to a reduction of mood disorders among children and young people from less privileged communities. The psycho-social aspects associated with this process, such as social support, increased self-confidence and self-efficacy, are to be emphasised [11].

In the light of available knowledge, the connection between physical activity and wellbeing among teenagers is a subject that is rarely addressed in the context of wider environmental determinants. Studies on the subject may lead to developing practical solutions, which may counteract the occurrence of the first symptoms of depression among young people.

\section{TOOLS AND QUESTIONNAIRES THAT HELP TO EVALUATE LOW MOOD AND ANXIETY AMONG TEENAGERS}

Therapy of mental disorders in young people uses a variety of tools to detect depression, frequently in the form of tests and questionnaires. These were developed for diagnostic purposes and are used in treatment. However, some may also be used in population studies.

Negative wellbeing symptoms are the most frequently identified among surveyed individuals. The first tool to be mentioned here is the Beck Depression Inventory (BDI) scale [12]. It assumes various forms depending on the number of questions used, from a short six-item version (BDI-6 short) [13] to various versions of a longer, 21-item scale (BDI-I, BDI-II) (21). The literature also describes the Mood and Feelings Questionnaire (MFQ). It is a 34-point questionnaire used for children and teenagers aged $8-18$ years to determine depression symptoms. The MFQ embraces affective, melancholy, somatic, and cognitive aspects of depression as well as those associated with suicidal thoughts. The individual is asked to define his or her feelings and mood over the last two weeks on a three-point scale [14].

The American Psychiatric Society developed a generally accepted test called the Diagnostic and Statistical Manual of Mental Disorders. Its DSM-III and DSM-IV versions are the most popular. In the latter, teenagers are asked about the presence of nine symptoms of depression from the previous month's perspective. These include questions concerning dysfunctions associated with bad physical wellbeing related to inability to cope with challenges at work or at school, personal hygiene, and the feeling of self-inefficacy [15]. Depression among young people may also be measured using the SMFQ scale - the Short Mood and Feelings Questionnaire. The index contains 13 questions aiming to rapidly assess worse wellbe- 
ing among children from the perspective of the previous two weeks [16].

International literature also widely describes a scale called the Centre for Epidemiologic Studies Depression Scale (CES-D), published by Laurie Radloff in 1977. The CES-D consists of 20 (or 10) questions addressing various depression symptoms that have occurred during the preceding week, most of them focusing on the affective component of depression $[17,18]$.

Clinical diagnosis is time consuming, while screen tests are very useful for a broader survey and may be used to study the general population of school children. They may take the form of short scales friendly for the respondents, used for self-assessment of mood and wellbeing, having a positive undertone. In this case the absence of positive emotions is an alarm signal for researchers. Textbooks for paediatricians promote the GHQ-12 and Kidscreen indexes [19], and more recently the WHO-5 scale is increasingly considered as useful.

The WHO-Five (WHO-5) is derived from a larger tool concerning the quality of life of patients suffering from diabetes [20]. The questionnaire was gradually reduced from 28 to 10 points, and subsequently to five questions (WHO-5). They relate to positive aspects such as wellbeing, vitality, and general satisfaction with life. The absence of positive emotions is an alarm signal treated as risk of depression. WHO- 5 has been translated into over 30 languages and is used in scientific research worldwide. In their broad survey of 213 studies using the WHO-5 test Topp et al. demonstrated that it may have very wide application. The WHO-5 works well for both genders, has a short and user-friendly form, and its validation has been confirmed [21].

\section{THE PURPOSE OF THE STUDY}

The purpose of the study was to analyse the occurrence of depression threat symptoms depending on the level of physical activity among young people, taking into account environmental factors associated with conditions growing up and the quality of social relationships.

The following research questions were formulated:

1. Does a high level of physical activity protect young people from depression?

2. Which environmental factors associated with the features of the family, place of residence, and the quality of social relationships show the strongest connection with depression threat symptoms in the studied group?

3. To what extent do the above environmental factors moderate the correlation between the level of physical activity and depression symptoms?

\section{MATERIAL AND METHODS}

The data are derived from the last round of international Health Behaviour in School-aged Children
(HBSC) studies performed in Poland during the school year $2017 / 2018$ on a representative group of 5225 school children. Children from 378 classes from 194 schools of various types from 16 provinces participated in an anonymous survey carried out in the schools. The response rate was $94.2 \%$ with regard to the randomly selected schools and $84.9 \%$ with regard to the number of students in the randomly selected classes. More detailed information about the study design and organisation of this round of HBSC studies is available in the national report [22].

\section{DEPENDENT VARIABLE}

The simple WHO-5 screen test was used, based on the results of the previously discussed scale of general well-being. The scale assumes values from 0 to 25 points. The level of eight points was adopted as the cut-off point for suspected depression symptoms. In the studied material the WHO-5 scale has a univariate structure (the main factor accounts for $58.2 \%$ of total variability) and a high level of reliability with Cronbach's $\alpha=0.816$.

\section{INDEPENDENT VARIABLES}

The prevalence of depression was studied according to the indicators related to the level of physical activity, socio-economic factors affecting social position, and social relationships. All the questions (unless noted otherwise) were sourced from the HBSC study protocol [23]. Only questions designed in the questionnaire for all age groups were chosen.

Four questions in the HBSC questionnaire referred to physical activity:

- MVPA - moderate-to-vigorous physical activity; the indicator enables us to establish on how many days in the preceding week young people devoted at least 60 minutes a day to physical activity (answers $0-7$ days);

- VPA - intensive physical activity outside school (seven answers from never to every day);

- Organised individual or team sports activities in free time (answers yes/no).

Five questions or measurement scales were related to socio-economic factors which affect social position:

- Academic achievement measured on a visual scale 0-10 points according to Goodman's concept (national question outside the HBSC protocol) [24];

- Family affluence according to the Family Affluence Scale (FAS) consisting of six questions; ranging from 0 to 13 points;

- Family structure in terms of the persons living with the respondent with the following response categories: living together with both biological persons, reconstructed family, being raised by a single parent, and other family situation;

- Place of residence in terms of the degree of urbanisation with four categories referring to the size of the 
residence location (question outside the international HBSC protocol);

- Feeling of safety in the area of residence, with four categories of answers from rarely or never to always.

Four combined indices and one single item referring to social relationships:

- The scale of coping in social situations consists of eight statements with five categories of answers from not at all to very well;

- The scale of peer support consists of four statements with seven categories of answers, from totally disagree to entirely agree;

- The scale of peer support consists of four statements with seven categories as above;

- The scale of social capital of the area of residence consists of four statements with five categories of answers from very much disagree to very much agree;

- Single item about school adjustment with four categories of answers, from don't like it at all to like it very much.

In addition, the gender of respondents and their age was taken into account, in three categories typical for HBSC studies: 11-12 years, 13-14 years, and 15-16 years.

An analysis of the psychometric features of the above scale indicates their univariate structure and satisfactory reliability, which was described in the technical report from the study [22].

\section{STATISTICAL ANALYSIS}

The above scales were re-coded into $2-5$ categories according to criteria applied in previous studies. The distribution in the sample was presented as well as the percentage of young people threatened with depression symptoms in each category. The odds ratio (OR) was estimated using logistic regression, with a $95 \%$ confidence interval, adjusting the analyses only for gender and age. Subsequently, a multivariate logistic regression model for risk of depression symptoms was estimated including all 16 independent variables. As a final step, as part of the analysis of the moderation effect, stratification for selected environmental factors was performed. In estimated

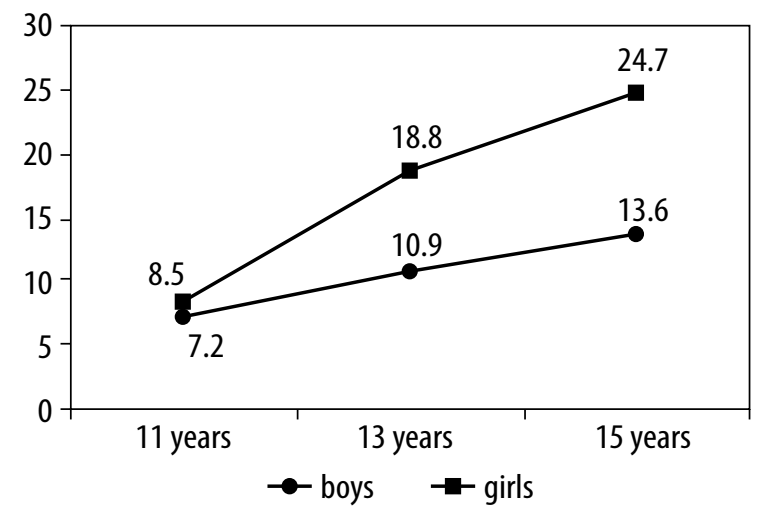

FIGURE 1. Symptoms of depression among school-aged children according to gender and age models the independent variables were as follows: the level of MVPA, gender, age, and the variables which were found to have the strongest impact in the previous step.

The data were analysed using the IBM SPSS v. 21 statistical package.

\section{RESULTS}

In the studied group of students aged 11-15 years symptoms of depression threat due to low mood were recorded in $14.2 \%$ of respondents $-10.6 \%$ in boys and $17.7 \%$ in girls $(p<0.001)$. The percentage increased in successive age groups, amounting to $7.9 \%$ among 11 -yearolds, $15.0 \%$ among 13 -year-olds, and $19.4 \%$ among 15 -year-olds $(p<0.001)$. The unfavourable difference for girls appeared in the two older age groups, while among 11 -year-olds it was statistically insignificant $(p=0.194)$ (Fig. 1).

Table 1 presents the prevalence of depression symptoms according to undertaken physical activity. With regard to all four variables, the protective effect of high physical activity was recorded, as well as rapid increase in lower mood symptoms in the case of deficit. The percentage of young people with these symptoms is $30 \%$ in case of very low MVPA and VPA level. The protective effect of organised sport activities also comes to light. If we look at relative risk measure, the protective effect of MVPA $(\mathrm{OR}=4.64)$ is observed.

Similarly, Table 2 presents the change in the percentages of young people threatened with depression symptoms in various social groups. With regard to all five analysed factors, a statistically significant connection was noted. The impact of perceived safety in the area of residence seems to be the strongest. The percentage of youths threatened with depression symptoms varies between $8.1 \%$ and $27.8 \%$ in comparison with extreme safety categories (OR =4.32). Another important factor is academic achievement, which is subject to strong social determinants in Poland. The percentage of young people with depression threat symptoms varies between $8.4 \%$ and $25.2 \%$ in the comparison between very good and very poor students. This is associated with the increase of the OR value to 3.54 . The effect of family affluence and structure as well as the level of urbanisation is significant, but the strength of the connection is much lower. Higher intensity of depression in large cities is an interesting observation.

Table 3 illustrates how the prevalence of depression symptoms among adolescents is distributed depending on the quality of social relationships. The impact of all five factors on depression threat symptoms is very strong. The threat increases systematically as the answers point to lower assessment in the family, school, and peer contacts categories. In the least privileged groups the percentages of young people with depressed mood oscillated around $30 \%$, while in the case of low family support they reached $38.3 \%(\mathrm{OR}=7.94)$. 
TABLE 1. Symptoms of depression in relation to physical activity

\begin{tabular}{|c|c|c|c|c|c|}
\hline \multirow{3}{*}{$\begin{array}{l}\text { Independent } \\
\text { variable }\end{array}$} & \multirow[t]{3}{*}{$n$} & \multicolumn{4}{|c|}{ Symptoms of depression according to WHO-5 } \\
\hline & & \multirow[t]{2}{*}{$\%$} & \multicolumn{3}{|c|}{ Logistic regression adjusted for age and gender } \\
\hline & & & $p$ & OR & $95 \% \mathrm{Cl}(\mathrm{OR})$ \\
\hline \multicolumn{6}{|l|}{ MVPA } \\
\hline Very low (0-1 day) & 518 & 32.35 & $<0.001$ & 4.64 & $3.39-6.36$ \\
\hline Low (2-4 days) & 2,301 & 15.20 & $<0.001$ & 1.80 & $1.36-2.37$ \\
\hline Moderate (5-6 days) & 1,484 & 10.34 & 0.167 & 1.24 & $0.91-1.67$ \\
\hline High & 891 & 7.83 & & 1.00 & \\
\hline \multicolumn{6}{|l|}{ VPA } \\
\hline Never & 376 & 31.35 & $<0.001$ & 4.25 & $2.97-6.08$ \\
\hline Once a month or less & 621 & 20.56 & $<0.001$ & 2.29 & $1.62-3.23$ \\
\hline Once a week & 828 & 16.13 & 0.001 & 1.81 & $1.29-2.53$ \\
\hline 2-6 times a week & 2,687 & 11.27 & 0.123 & 1.27 & $0.94-1.72$ \\
\hline Every day & 683 & 8.25 & & 1.00 & \\
\hline \multicolumn{6}{|l|}{ Team sports } \\
\hline No & 3,033 & 17.94 & $<0.001$ & 1.91 & $1.59-2.20$ \\
\hline Yes & 2,139 & 8.98 & & 1.00 & \\
\hline \multicolumn{6}{|l|}{ Individual sports } \\
\hline No & 3,801 & 15.52 & $<0.001$ & 1.47 & $1.21-1.79$ \\
\hline Yes & 1,360 & 10.72 & & 1.00 & \\
\hline
\end{tabular}

MVPA - moderate-to-vigorous physical activity, VPA - intensive physical activity outside school

In the multivariate logistic regression model family support, self-efficacy in social relations, and school adjustment proved to be the strongest predictors of depression symptoms. The final model contains 12 explanatory variables, has a Cox \& Snell R-sq. coefficient of 0.273 , and a Hosmer-Lemeshow goodness of fit test significance of $p=0.021$. After adjustment for the effect of all the factors, relative risk indicators are obviously lower than those presented in Table 1-3 and fluctuate between 1.55 (social capital of the neighbourhood) and 3.20 (family support). In general, participation in individual sports, material resources of the family, family structure, and place of residence did not qualify for the final model. It did, however, include three variables associated with physical activity, including MVPA - having the greatest impact (Fig. 2). In multivariate models estimated for boys and girls the strong effect of MVPA is maintained, and among boys it is the third most important factor.

When estimating the series of specific models for subgroups of young people it was demonstrated that variables related to living conditions moderate the correlation between physical activity and depression symptoms, despite not being included in the final model. The strongest protective effect of physical activity is particularly visible in the least affluent families, in single parent families, and in large cities (Table 4).

\section{DISCUSSION}

The study presents data concerning a representative group of 5225 schoolchildren aged 11-15 years. Sixteen potential determinants of mood disorders among teenagers were analysed, including four associated with the level of physical activity. The multivariate logistic regression model confirmed that the protective effect of physical activity was maintained, although the impact of the quality of social relationships proved to be dominating.

The authors of the study demonstrated that failure by young people to comply with the recommended level of physical activity may lead to lowered mood. This is confirmed by cross-sectional surveys of British youth ( $n=2951)$ conducted by Wiles et al., which demonstrated the specific influence of regularity in participation in sport activities [25]. In his literature review Janssen came to similar conclusions. In order to improve the health of young people, physical activity ought to be at least of moderate intensity, but intensive, regular exercise unquestionably gives the greatest benefits [26]. The type of physical activity and its impact on the mood of Canadian teenagers was analysed by Boone et al. Similarly to the authors of this article, he demonstrated that participation in team sports is the best protective measure in mood disorders among teenagers because it provides the opportu- 
TABLE 2. Symptoms of depression in relation to social position and living conditions

\begin{tabular}{|c|c|c|c|c|c|}
\hline \multirow{3}{*}{$\begin{array}{l}\text { Independent } \\
\text { variable }\end{array}$} & \multirow[t]{3}{*}{$n$} & \multicolumn{4}{|c|}{ Symptoms of depression according to WHO-5 } \\
\hline & & \multirow[t]{2}{*}{$\%$} & \multicolumn{3}{|c|}{$\begin{array}{l}\text { Logistic regression } \\
\text { adjusted for age and gender }\end{array}$} \\
\hline & & & $p$ & OR & $95 \% \mathrm{Cl}(\mathrm{OR})$ \\
\hline \multicolumn{6}{|l|}{ School achievements } \\
\hline Poor (0-4) & 827 & 25.19 & $<0.001$ & 3.54 & $2.65-4.71$ \\
\hline Average (5-6) & 1,720 & 14.31 & $<0.001$ & 1.75 & $1.33-2.30$ \\
\hline Good (7-8) & 1,687 & 12.00 & 0.008 & 1.46 & $1.10-1.93$ \\
\hline Very good $(9-10)$ & 931 & 8.42 & & 1.00 & \\
\hline \multicolumn{6}{|l|}{ Family affluence } \\
\hline Very poor (0-4) & 383 & 18.75 & 0.001 & 1.70 & $1.23-2.34$ \\
\hline Rather poor (5-6) & 1,150 & 15.32 & 0.026 & 1.31 & $1.03-1.67$ \\
\hline Average (7-9) & 2,365 & 13.84 & 0.225 & 1.14 & $0.92-1.41$ \\
\hline Well-off (10-13) & 1,188 & 12.80 & & 1.00 & \\
\hline \multicolumn{6}{|l|}{ Family structure } \\
\hline Both biological parents & 4,013 & 13.22 & & 1.00 & \\
\hline Reconstructed & 241 & 17.87 & 0.152 & 1.29 & $0.91-1.84$ \\
\hline Single parent & 839 & 17.35 & 0.001 & 1.61 & $1.14-1.73$ \\
\hline Other & 132 & 18.11 & 0.044 & 1.45 & $1.01-2.59$ \\
\hline \multicolumn{6}{|l|}{ Urbanicity } \\
\hline Big city $(>100,000)$ & 1,202 & 16.13 & 0.028 & 1.26 & $1.03-1.55$ \\
\hline Average size city & 650 & 15.23 & 0.139 & 1.21 & $0.94-1.57$ \\
\hline Small town $(<50,000)$ & 1,254 & 14.54 & 0.068 & 1.22 & $0.99-1.50$ \\
\hline Rural areas & 2,094 & 12.62 & & 1.00 & \\
\hline \multicolumn{6}{|c|}{ Safety in the neighbourhood } \\
\hline Rarely or never & 122 & 27.83 & $<0.001$ & 4.32 & $2.77-6.74$ \\
\hline Sometimes & 407 & 28.07 & $<0.001$ & 3.93 & $2.99-5.16$ \\
\hline Most of the time & 2,369 & 16.84 & $<0.001$ & 1.96 & $1.62-2.37$ \\
\hline Always & 2,254 & 8.05 & & 1.00 & \\
\hline
\end{tabular}

nity to integrate with peers and build positive, supportive relationships with coaches [27].

Literature points to the fact that during adolescence young people often come into conflict with their parents and their surroundings. In Iceland a study was carried out by Sigfusdottir et al. on a sample of seven thousand teenagers. He demonstrated that depressive behaviours of young people are exacerbated by family conflict, while physical activity may reduce the tension between parents and children [28]. In the currently discussed Polish study a supportive family environment proved to be the main factor protecting young people against depressive conditions.

The relationship between physical activity and depression symptoms among teenagers ought to be analysed in the context of the factors associated with the environment in which they grow up. The impact of family, school, and neighbourhood ought to be analysed separately. Literature devotes a great deal of attention to family affluence, which in our research was proven to be a moderator of the correlation between physical activity and mental health. Programs aimed at providing equal opportunities to children from less affluent families ought to promote the participation of teenagers in sports activities. Holt et al. demonstrated that physical activity plays a protective role in the mental health of less affluent families [29]. Tomé et al. also pointed to the macroeconomic determinants of wellbeing and depression symptoms among youths [10]. The second factor related to the family environment, which is taken into account in the context of the influence of physical activity on the mental health of young people, is family structure. Divorced parents, single mothers, and the appearance of a stepfather or stepmother may create stronger depression symptoms among teen- 
TABLE 3. Symptoms of depression in relation to social bonds

\begin{tabular}{|c|c|c|c|c|c|}
\hline \multirow{3}{*}{$\begin{array}{l}\text { Independent } \\
\text { variable }\end{array}$} & \multirow[t]{3}{*}{$n$} & \multicolumn{4}{|c|}{ Symptoms of depression according to WHO-5 } \\
\hline & & \multirow[t]{2}{*}{$\%$} & \multicolumn{3}{|c|}{ Logistic regression according to age and gender } \\
\hline & & & $p$ & OR & $95 \% \mathrm{Cl}(\mathrm{OR})$ \\
\hline \multicolumn{6}{|l|}{ Social self-efficacy } \\
\hline Very low $(0-15)$ & 897 & 30.06 & $<0.001$ & 6.98 & $5.05-9.65$ \\
\hline Rather low (16-21) & 1,695 & 14.54 & $<0.001$ & 2.70 & $1.96-3.72$ \\
\hline Average (22-26) & 1,521 & 9.67 & 0.002 & 1.72 & $1.23-2.40$ \\
\hline High (27-32) & 865 & 5.83 & & 1.00 & \\
\hline \multicolumn{6}{|l|}{ Peer social support } \\
\hline Very low (0-6) & 840 & 29.51 & $<0.001$ & 6.41 & $4.71-8.72$ \\
\hline Rather low (7-13) & 1,491 & 16.37 & $<0.001$ & 2.97 & $2.20-4.04$ \\
\hline Average (14-20) & 1,892 & 9.75 & 0.004 & 1.56 & $1.15-2.12$ \\
\hline High (21-24) & 936 & 6.52 & & 1.00 & \\
\hline \multicolumn{6}{|l|}{ Family social support } \\
\hline Very low $(0-10)$ & 702 & 38.32 & $<0.001$ & 7.94 & $6.00-10.50$ \\
\hline Rather low (11-17) & 1,266 & 16.59 & $<0.001$ & 2.74 & $2.08-3.61$ \\
\hline Average (18-23) & 1,780 & 9.28 & 0.004 & 1.50 & $1.14-1.99$ \\
\hline High (24) & 1,382 & 5.97 & & 1.00 & \\
\hline \multicolumn{6}{|c|}{ Neighbourhood social capital } \\
\hline Very low (0-9) & 859 & 26.48 & $<0.001$ & 4.72 & $3.46-6.45$ \\
\hline Rather low (10-13) & 1,828 & 16.69 & $<0.001$ & 2.65 & $1.97-3.56$ \\
\hline Average (14-16) & 1,386 & 9.12 & 0.033 & 1.43 & $1.03-1.97$ \\
\hline High (17-20) & 998 & 5.93 & & 1.00 & \\
\hline \multicolumn{6}{|l|}{ Liking school } \\
\hline Don't like it at all & 522 & 31.84 & $<0.001$ & 5.80 & $4.39-7.66$ \\
\hline Don't like it very much & 968 & 21.84 & $<0.001$ & 3.41 & $2.64-4.40$ \\
\hline Like a bit & 2,156 & 11.82 & $<0.001$ & 1.74 & $1.37-2.22$ \\
\hline Like a lot & 1,564 & 6.80 & & 1.00 & \\
\hline
\end{tabular}

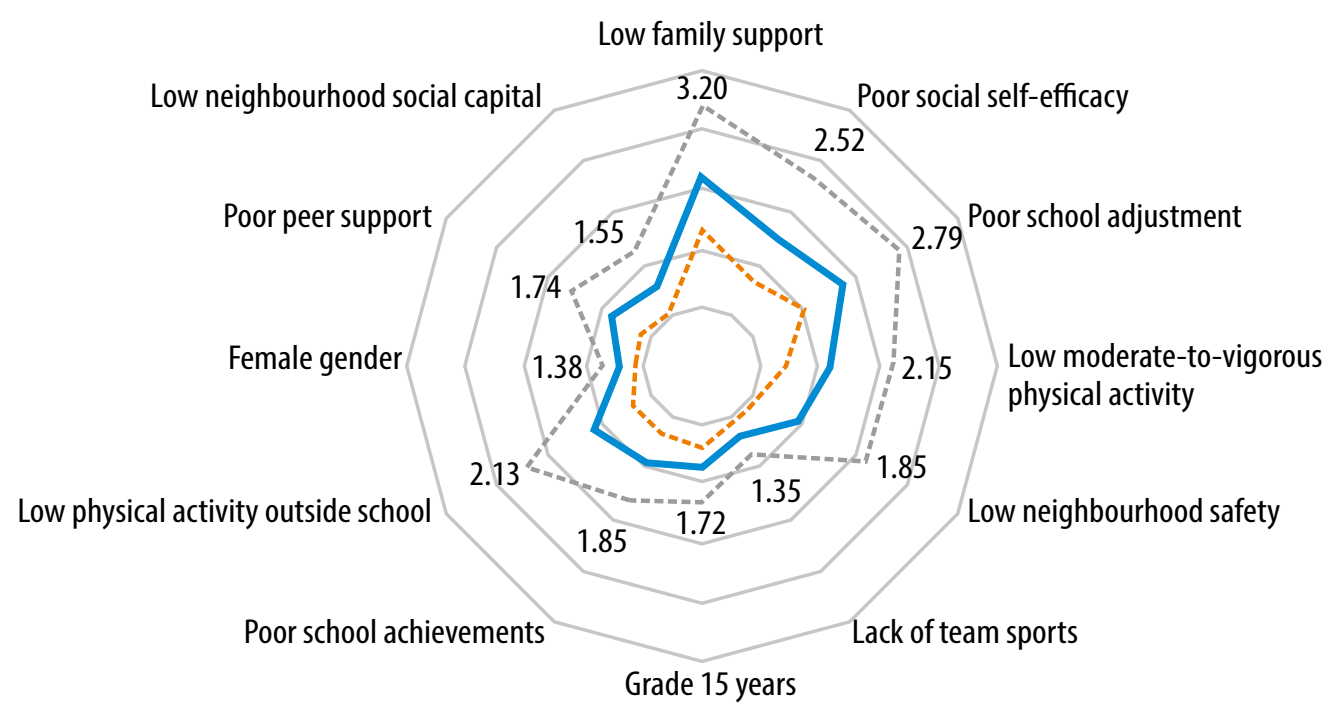

FIGURE 2. Independent depression predictors - multivariate regression (OR) 
TABLE 4. Relative risk (OR) of depression symptoms according to WH0-5 related to low moderate-to-vigorous physical activity (MVPA) moderated by selected social factors

\begin{tabular}{|l|c|c|c|c|}
\hline \multirow{3}{*}{ Moderator* } & The worst level & Second level & Third level & The best level \\
\cline { 2 - 5 } & $\begin{array}{c}\text { OR } \\
95 \% \text { CI (OR) }\end{array}$ & $\begin{array}{c}\text { OR } \\
95 \% \text { Cl (OR) }\end{array}$ & $\begin{array}{c}\text { OR } \\
95 \% \text { CI (OR) }\end{array}$ & $\begin{array}{c}\text { OR } \\
95 \% \text { CI (OR) }\end{array}$ \\
\hline Family affluence & 6.34 & 4.97 & 3.69 & 4.57 \\
\cline { 2 - 5 } & $(3.23-11.75)$ & $(2.33-10.60)$ & $(2.15-6.35)$ & $(2.19-9.54)$ \\
\hline Family structure & 3.32 & 5.20 & 2.72 & 4.09 \\
\cline { 2 - 5 } & $(0.32-34.38)$ & $(2.23-12.13)$ & $(0.63-11.81)$ & $(2.74-6.12)$ \\
\hline Urbanicity & 8.82 & 6.72 & 2.27 & 3.38 \\
\cline { 2 - 5 } & $(3.54-18.14)$ & $(2.53-11.84)$ & $(1.47-5.24)$ & $(1.90-6.02)$ \\
\hline
\end{tabular}

Comparing MVPA 0-1 days to MVPA 7 days adjusted for gender, grade, family support, social self-efficacy, school satisfaction. * Subsequent levels correspond to: family affluence - very low, rather low, average, high; family structure - other or unknown, single parent, step parent, both biological parents; urbanicity - big cities, medium-size cities, small cities, rural areas

agers. Physical activity, on the other hand, may moderate this correlation. This was confirmed by Chinese researchers [30] who analysed the impact of physical activity on the mental health of young people, taking into account the family structure $(n=5504)$, as well as Swiss researchers in a comprehensive survey of international literature [31]. Physical activity may also alleviate depression syndromes among children threatened with social alienation. As indicated by the studies of Petty et al., it is particularly effective among children from racial minorities and obese children [32].

The place of residence may be connected with the risk of occurrence of first depression symptoms or ill-being among teenagers. In an Australian study Kremer et al. demonstrated that children from large cities are more prone to depression [33]. This observation was also confirmed by Romer et al. from the US, who showed that young people from large cities, especially from low economic status areas, are more prone to mood disorders [34]. The unfavourable effect of the urban environment is confirmed by the research described in this study.

\section{LIMITATIONS OF THE STUDY}

The present study has some limitations. The employed WHO-5 questionnaire is a tool with a positive undertone, and it is relatively soft in comparison with the most popular tests, as described earlier, used to detect depression. The short form of the scale, which may be used in multifaceted questionnaires, is one of its strengths. The WHO-5 may be used for younger adolescents, such as the 11-year-old respondents. The present study is of cross-sectional nature, so it is difficult to speak about a cause and effect relationship. Nonetheless it should be emphasised that the investigations involved a large, representative sample and took into account a rarely encountered large number of diverse factors and their mutual interactions. Their value is increased by the connection with generally recognised HBSC studies.

\section{CONCLUSIONS}

Intensive physical activity protects young people aged 11-15 years from depression symptoms. Its protective effect is maintained after taking into account various other predictors of low mental wellbeing.

Poor quality of family relationships, inability to cope in social situations, and a negative attitude to school have a particularly strong impact on increased risk of depression symptoms.

Factors associated with living conditions do not have a direct influence on depression risk but moderate the effect of physical activity on its symptoms. The protective effect of physical activity is particularly visible in single-parent families, in poor families, and in large cities. It is important to design programs providing equal opportunities to children growing up in a less favourable environment by providing young people from the poorest families with the ability to participate in free-of-charge sports activities, particularly team sports.

\section{DISCLOSURE}

The authors declare no conflict of interest.

\section{REFERENCES}

1. Snyder M. Changing the tide: stigma, school youth, and mental illness. NASN Sch Nurse 2015; 30: 130-132.

2. Horwitz SM, Leaf PJ, Leventhal JM, et al. Identification and management of psychosocial and developmental problems in community-based, primary care pediatric practices. Pediatrics 1992; 89: 480-485.

3. Katon WJ, Richardson L, Russo J, et al. Quality of mental health care for youth with asthma and comorbid anxiety and depression. Med Care 2006; 44: 1064-1072.

4. Masten AS, Roisman GI, Long JD, et al. Developmental cascades: linking academic achievement and externalizing and internalizing symptoms over 20 years. Dev Psychol 2005; 41: 733-746.

5. Bickham DS, Hswen Y, Rich M. Media use and depression: exposure, household rules, and symptoms among young adolescents in the USA. Int J Public Health 2015; 60: 147-155. 
6. Costello EJ, Angold A, Burns BJ, et al. The Great Smoky Mountains Study of Youth. Goals, design, methods, and the prevalence of DSM-III-R disorders. Arch Gen Psychiatry 1996; 53: 1129-1136.

7. Katon W, Richardson L, Russo J, et al. Depressive symptoms in adolescence: the association with multiple health risk behaviors. Gen Hosp Psychiatry 2010; 32: 233-239.

8. Murray K, Rieger E, Byrne D. Body image predictors of depressive symptoms in adolescence. J Adolesc 2018; 69: 130-139.

9. Przybylski AK, Bowes L. Cyberbullying and adolescent well-being in England: a population-based cross-sectional study. Lancet Child Adolesc Health 2017; 1: 19-26.

10. Tomé G, Gaspar de Matos M, Camacho I, et al. Impact of Alienation on Portuguese Adolescents' Well-being. J Psychol Psychother 2016, 6: 5 .

11. Bélair M-A, Kohen DE, Kingsbury M, Colman I. Relationship between leisure time physical activity, sedentary behaviour and symptoms of depression and anxiety: evidence from a population-based sample of Canadian adolescents. BMJ Open 2018; 8: e021119.

12. Henkel V, Mergl R, Kohnen R, et al. Identifying depression in primary care: a comparison of different methods in a prospective cohort study. BMJ 2003; 326: 200-201.

13. Beck AT, Ward $\mathrm{CH}$, Mendelson $\mathrm{M}$, et al. An inventory for measuring depression. Arch Gen Psychiatry 1961; 4: 561-571.

14. Angold A, Costello EJ, Pickles EJ, Winder F. The development of a questionnaire for use in epidemiological studies of depression in children and adolescents. Medical Research Council, London 1987.

15. Vahia VN. Diagnostic and statistical manual of mental disorders 5 quick glance. Indian J Psychiatry 2013; 55: 220-223.

16. Angold A, Costello EJ, Messer SC, et al. Development of a short questionnaire for use in epidemiological studies of depression in children and adolescents. Int J Methods Psychiatr Res 1995; 5: 237 249.

17. Radloff LS. The CES-D Scale: A Self-Report Depression Scale for Research in the General Population. App Psychol Meas 1977; 1: 385-401.

18. Faulstich ME, Carey MP, Ruggiero L, et al. Assessment of depression in childhood and adolescence: an evaluation of the Center for Epidemiological Studies Depression Scale for Children (CES-DC). Am J Psychiatry 1986; 143: 1024-1027.

19. Mazur J, Tabak I. Narzędzia do badań przesiewowych w wykrywaniu zaburzeń zdrowia psychospołecznego dzieci i młodzieży. In: Profilaktyka w pediatrii, Woynarowska B (ed.). PZWL, Warszawa 2008; 324-331.

20. Bech P, Gudex C, Johansen KS. The WHO (Ten) Well-Being Index: validation in diabetes. Psychother Psychosom 1996, 65: 183-190.

21. Topp CW, Østergaard SD, Søndergaard S, Bech P. The WHO-5 Well-Being Index: A Systematic Review of the Literature. Psychother Psychosom 2015; 84: 167-176.

22. Mazur J, Małkowska-Szkutnik A (eds.). Zdrowie uczniów w 2018 roku na tle nowego modelu badań HBSC. Instytut Matki i Dziecka, Warszawa 2018.

23. Inchley J, Currie D, Cosma A, Samdal O (eds.). Health Behaviour in School-aged Children (HBSC) Study Protocol: background, methodology and mandatory items for the 2017/18 survey. CAHRU, St Andrews 2018.

24. Goodman E, Huang B, Schafer-Kalkhoff T, Adler NE. Perceived socioeconomic status: a new type of identity that influences adolescents' self-rated health. J Adolesc Health 2007; 41: 479-487.

25. Wiles NJ, Haase AM, Lawlor DA, et al. Physical activity and depression in adolescents: cross-sectional findings from the ALSPAC cohort. Soc Psychiatry Psychiatr Epidemiol 2012; 47: 1023-1033.

26. Janssen I, LeBlanc A. Systematic review of the health benefits of physical activity and fitness in school-aged children and youth. Int J Behav Nutr Phys Act 2010; 7: 40.
27. Boone E, Leadbeater B. Game on: diminishing risks for depressive symptoms in early adolescence through positive involvement in team sports. J Res Adolesc 2006; 16: 79-90.

28. Sigfusdottir ID, Asgeirsdottir BB, Sigurdsson JF, Gudjonsson GH. Physical activity buffers the effects of family conflict on depressed mood: a study on adolescent girls and boys. J Adolesc 2011; 34: 895-902.

29. Holt N, Kingsley B, Tink L, Scherer J. Benefits and challenges associated with sport participation by children and parents from low-income families. Psychol Sport Exerc 2011; 12: 490-499.

30. Cao H, Qian Q, Weng T, et al. Screen time, physical activity and mental health among urban adolescents in China. Prev Med 2011; 53: 316-320.

31. Michaud P-A, Jeannin A, Suris J. Correlates of extracurricular sport participation among Swiss adolescents. Eur J Pediatr 2006, 165: 546-555.

32. Petty KH, Davis CL, Tkacz J, et al. Exercise effects on depressive symptoms and self-worth in overweight children: a randomized controlled trial. J Pediatr Psychol 2009; 34: 929-939.

33. Kremer P, Elshaug C, Leslie E, et al. Physical activity, leisure-time screen use and depression among children and young adolescents. J Sci Med Sport 2014; 17: 183-187.

34. Romer D, Bagdasarov Z, More E. Older versus newer media and the well-being of united states youth: results for national longitudinal panel. J Adolesc Health 2013; 52: 613-619. 